\title{
Microbiological

\section{Intestinal microbiome and related diseases: A recent updated review}

\author{
Fatemeh Bagheri ${ }^{* 1,2}$ and Davood Zare ${ }^{1}$ \\ ${ }^{1}$ Department of Biotechnology, Iranian Research Organization for Science and Technology, Tehran, Iran \\ ${ }^{2}$ Islamic Azad University, Pharmaceutical Science Branch,Department of Microbiology, Tehran, Iran
}

\begin{abstract}
The set of bacteria, viruses and fungi that live on human bodies, is called in all microbiota and its genes are called microbial. Microbial, because of its products, its response to the environment and its reciprocal response to other systems is considered as one of the organs of the body. Sometimes, it is also called the second genome of human beings and the microbial genes that form microbial are more than 1\% of human beings' genes and in bowel there are more than three million bacterial genes. One of the most important parts of body that its microbial has attracted the attention of scientists very much is the microbial of alimentary canal. A set of unicellular organisms in our bowel, called intestinal microbial appears as the important index of health. Errors and mistakes in a suitable microbial colonization in the early life causes food sensitivity, allergic responses, type 1 diabetes and other disorders of autoimmune. The important role of the intestinal microbiota in weight adjustment is through harvest and appetite control. Intestinal microbiota has an important role in the pathology of various inflammatory bowel diseases and also developing colorectal, gastric and prostate cancers and cardio metabolic disorders.
\end{abstract}

KEY WORDS: GUT MICROBIOME, INSULIN RESISTANCE, PROBIOTICS, BIFIDOBACTERIUM

\section{INTRODUCTION}

The set of bacteria, viruses and fungi that live on human bodies, is called in all microbiota and its genes are called microbial ( Microbial, because of its products, its response to the environment and its reciprocal response to other systems is considered as one of the organs of the body. Sometimes, it is also called the second genome of human beings and the microbial genes that form microbial are more than 1\% of human beings' genes and in bowel there are more than three million bacterial genes, (Petersen et al. 2017, Greenhalgh et al. 2016 Miliani et al. 2018).

\section{ARTICLE INFORMATION:}

*Corresponding Author: bagherizohal1 @yahoo.com Received $2^{\text {nd }}$ Jan, 2018

Accepted after revision $13^{\text {th }}$ March, 2018

BBRC Print ISSN: 0974-6455

Online ISSN: 2321-4007 CODEN: USA BBRCBA

- Thomson Reuters ISI ESC / Clarivate Analytics USA and

W. Crossref Indexed Journal

NAAS Journal Score 2017: 4.38 SJIF 2017: 4.196

- A Society of Science and Nature Publication, Bhopal India 2018. All rights reserved.

Online Contents Available at: http//www.bbrc.in/

DOI: $10.21786 / \mathrm{bbrc} / 11.1 / 13$ 
One of the most important parts of body that its microbial has attracted the attention of scientists very much is the microbial of alimentary canal. Human being's alimentary canal is an organized microbial composition that carries more than ten times of human being's cells (Vinke et al. 2017). Therefore, intestinal microbiome is considered as the mammalian super organism, (Arboleya et al. 2016).Genetic, the birth method, nutrition patterns of infants and taking antibiotics, the living health conditions and dietary habits for a long time have all important rolls in the formation of the intestinal microbes, (Caesar et al. 2016). However, it seems that alimentary canal of every mature person might have a unique and slightly stable microbiota. The early researches about the analysis of ribosomal DNA 16S, are used to determine and count the distal and fecal intestinal microbiota. More than $90 \%$ of the bacterial phenotypes that exist in the intestinal microbiota in healthy human beings, are three bacterial classes that include Bacterioides, Furmicut and Actinobacteria (Haghes et al. 2017).

In the early years of 1900 s, two Nobel Prizes in physiology and medical science were offered to the scientists that determined the relation between microbes and human health. The first Nobel Prize was offered to Robert Koch that linked microbes to infectious diseases, while the second prize was offered to Ilya Mechnikov who, for the first time, used the alive micro-organisms to protect human health. Now a complicated set of relations between microbiota and human beings has been determined (Vinke et al. 2017).

The microbial has an important role in the formation of human being's characteristics. For example, one of the roles of intestinal microbial flora is its effect on the obesity. The fundamental metabolic mechanisms of this resistance include the absorption of glucose and the production of a short chain of fatty acids from duct and the decrease of lipogenesis related to the liver, the decrease of fatty acid oxidation, the decrease of accumulation of triglycerides in adipocytes (Sicard et al. 2017) and also the intestinal microbiota has an important role in food digestion and in the host immunologic response (Dominianni et al. 2016).

\section{DISEASES AND INTESTINAL MICROBES}

Necrotizing Enter colitis: The growth of intestinal microbial in the early life and its importance in infants who are at risk for developing necrotizingenter colitis have been determined. The intestinal microbial of the infants with NEC was determined by the difference in the microbial composition such as the increase in frequency of Gamma-proteobacteria that is a common feature of intestinal microbial in the condition of disease (Timothy et al. 2016).

The difference in the microbial composition in fecal microbiota is the first step to develop NEC. Probiotics or the method of nutrition can change the microbial composition into an intestinal microbial, resistant to the disease. In order to decrease the outbreak of NEC in premature infants, a diet that consists of breast milk has been recommended and the milk supplement given by mother has been considered as a way to prevent NEC. In addition to milk supplement, several probiotics have been successful in preventing the severe NEC and decreasing the mortality of premature infants for any reason, including infants with low weights at birth. The recent investigations about the infants with NEC, have shown the increase in frequency of Proteobacteria such as Citrobacter in fecal microbiota. Besides, Versalovic and et al (2017), have recently reported a more ratio of Gamma-proteobacteria in fecal microbiota before the NEC diagnosis in infants. Healthy diets for NEC include taking non-edible (injectable) antibiotics for a long time that can decrease the intestinal microbial diversity and prevent colonization by a variety of microbes (Versalovic et al. 2017).

The Infection of Clostridium Diphyles and Acute Gastroenteritis: Ecological and microbial disorders may be cured by the use of anti-microbial medicines and chemotherapy and can be improved by probiotics. In the last decade, outbreak of the disease along with Clostridium diphylesin infants has increased. Gorbach and his colleagues have shown the successful treatment of this disease by the use of Lactobacillus rhamnosus strain taken from human beings. This strain is usually known under the title of LGG probiotic. Several investigations about infants have shown that probiotics may be effective for suppressing diarrhea along with antibiotic; and probiotics may increase the maintenance of microbial diversity as a mechanism for soothing the disease phenotype (Johnson et al. 2016).

Irritable Bowel Syndrome: The difference in composition or intestinal dissbiosis microbial in irritable bowel syndrome was reported in 2011, and the effects of the disease based on the differences in bacterial composition were discovered. The increase of Gamma-proteobacteria in adult children with IBS and its relation with a group containing intestinal pathogens was accompanied by the increase of pains; and it explained the decrease of Bifidobacterium classes and Verrucomicrobium in the infants with IBS diarrhea. The consumption of Bifidobacterium in adults with IBS decreased the symptoms and determined the possible advantage of probiotic treatments in IBS with probiotic strains which have been selected carefully. The significance of intestinal Bifidobacterium 
may explain the relative success of compound solution of Bifidobacterium - lactobacillus (Johnson et al. 2016).

Inflammatory Bowel Disease: The difference in the intestinal microbial composition in different investigations on patients with colon disease and wounded colitis has been reported (Johnson et al. 2016). This imbalance and disorder in the host microbiota, called dissbiosis, can form the inflammatory response by the host. Such differences include the decrease of bacterial fila ratios of Bacteroides and Freiquito and the development of Protobacterium filum in patients with inflammatory bowel disease. The relative increase of Proteobacteria and Gamma-proteobacteria in recent investigations emphasizes on the possible significance of gram-negative bacteria in adults and IBD of infant. The particular components of Gamma-proteobacteria help diagnose the infants with IBD; a special example is the increase of E.coli and Shigella classes in the infants with ulcerative colitis, (Johnson et al. 2016).

Celiac Disease: Although the microbial composition of the small intestine in patients with celiac disease is not different, differences in intestinal microbial in stool samples, collected from the patients with celiac disease, have been determined. The equivalent changes have been reported in stool microbial and stool and urine metabolism in the infants with celiac disease, in comparison with healthy controls. In the PROFICEL investigation, it was determined that breast feeding with its possible "feeder" effects on the useful microbes has an influence on the relative talent to develop celiac disease. In a report, the relative timing of the arrival of gluten in early childhood had an influence on the relative talent to develop the disease and disease phenotypes were improved by the changes in intestinal microbial and metabolism (Timothy et al. 2016).

An interesting aspect of celiac disease was the presence of the bacterial metabolizing gluten, such as rothia in edible microbial. Gluten metabolizing microbes in oral or intestinal microbial may decrease the relative talent of people with genetic background to the celiac disease. The probiotic solutions in the future may include the following issues: paying attention to the probiotics with gluten metabolism genes and the probiotic ability to increase the function of gluten metabolizing bacteria in microbial (Johnson et al. 2016).

The Effect of Microbial on Depression: Just in a few numbers of investigations, the anxiety effects of probiotics in clinical trials have been investigated. In a research, the positive effects of lactobacillus probiotics helveticus R0052 and lactobacillus B.longum R0175 in an animal model were approved in adult volunteers in a random two-way blind trial. The active treatment decreased psychological distress and subsequently, decreased the urinary cortisol (Johnson et al. 2016).

The Role of Microbiota in the Autistic Spectrum Disorders (ASD): ASD is a group of evolutionary neurological disorders that includes autism, childhood disruptive disorder and inclusive growth disorder. ASDs along with disabilities, include social retirement, speech disorder and repetitive behavior. Gastrointestinal (GI) disorders in infants with autism are very common and it has been shown that the number of GI symptoms is related to autism very much. Particularly, Bacteroides in patients with autism increased significantly, while in people watching the pyromicots were more. On the contrary, in another investigation, patients with autism and (ASD + GI) GI problems were compared with patients with only GI problems and it was shown that Bacteroides decreased and pyromicots increased and there were Betaproteobacteria (Sutarla) in patients with ASD + GI (Greenhalgh et al. 2016).

Etiology of ASD is very complicated and multifaceted and includes gene, environment and diet; and besides, it has been shown that intestinal microbes link to the brain through the intestine-brain axis in order to improve the growth of brain and the function and behavior with the brain. The recent evidence shows that intestinal microbes influence the development of central neurological system and the response to the stress (Bailey et al. 2015).

The Intestine-brain Axis in Health and Disease: The term, "intestine-brain" or "brain-intestine" axis is used increasingly to define a neurohumoral two-way communication system. It consists of neural paths and humoral paths that include cytokine, hormones and neuropeptides as signal molecules. The axis brain-intestine and intestine-brain microbes includes central neurological system, the nerves and glands and neurological immune system, sympathetic and parasympathetic nerves of automatic neurological system, the intestinal neurological system and intestinal microbes. By the use of this two-way communication network, the received signals from the brain can influence the secretion of the alimentary canal and mutually, visceral messages from the alimentary canal can influence the function of brain. Intestinal microbes through the intestine-brain axis are related to the function of brain and also neurological diseases, (Dianan and Cryan, 2017)

Tiny Bowel Habitats and (ASD): Bolte et al (2016) have published the relation of ASD and low degree intestinal infection with Clostridium tetani. This bacterium belongs to the piermicots branch. They are mandatory anaerobic rod-shape. Some of the most important 
biological pathogens that belong to this kind of bacterium are Clostridium botulinum, Clostridium diphyles and Clostridium tetani. These species are known for the production of neurotoxin and the poison produced by Clostridium tetani in the intestines of laboratory animals can be transferred to the central neurological system through vagus nerve and leads to disorder in neurons. It has been shown that this kind of inhibition may lead to the thousands of behavioral defects observed in the infants with ASD. They made investigations on the infants with autism and in the vancomycin treated infants they showed the improvement of digestive problems such as stomachache, constipation or diarrhea. Besides, the behavioral developments were observed by the considerable decrease in the aggression, the increase of eye contact and remarkable improvement in speech. However, these changes depended on vancomycin treatment and all the symptoms relapsed after they stopped taking antibiotics, (Bolte et al. 2016).

The research done by Sandler et al (2015), has provided one of the first obvious scientific documentations on the link between the intestine-brain axis and ASD. Fine gold and et al (2016), investigated the feces of infants with ASD and compared it with the control group that confirmed the above-mentioned results. Parracho and et al (2014), stated that the comparison of the feces of infants with ASD with the healthy brothers and sisters and the unrelated healthy control group showed that the infants with ASD had higher levels of some species of clostridium. Besides, control groups with normal growth and infants with ASD and sibling with normal function showed that infants with ASD had higher levels of Bacteroides and lower levels of piermicots than the control group, (Timothy et al. 2016).

Besides, the infants with ASD showed a lower level of some species of Bifidobacterium while Desulfovibrio was higher. The research done by Vang and et al (2014), showed a lower level of Bifidobacterium in the infants with ASD as well. In fact, the research done by Finegold and et al (2016), showed that there was Desulfovibrio in half of the people with autism and some siblings. More interestingly, none of the control people had Desulfovibrio. It was known that the infants with ASD don't have enough sulphur. Aldred and et al (2014), showed that the people with autism, in comparison to the healthy people, have lower levels of plasma sulfate but a considerable higher levels of urinary sulfate (Haghes et al. 2017).

The presence of revitalizing sulfate bacteria such as Desulfovibrio in the infants with ASD can be one of the observed disorders in the sulphur metabolism. Besides, the intensity of ASD behavior has a positive relationship with the increase of Desulfovibrio species and was also known that there were Hemophilus parainfluenza bacteria in the patients with IBS with more frequency.
The recent investigations on microbiota in adults with different sub-types of IBS also confirmed the bacterial dissbiosis specific to the intestineand in the patients with IBS-D, Bifidobacteria decreased and Gamma-proteobacteria increased (Greenhalgh et al. 2016). Metabolomics in ASD investigation showed that the infants with autism had abnormal levels of urinary hypores, hypo-hydroxy-4 and N-methyl-2-pyridon-5-carboxamide (2PY) than the control group.

The blood plasma of the patients with autism showed the increase in most of saturated fatty acids, except propionic acid and the decrease in most of unsaturated fatty acids that can be related to the several processes including oxidative stress to disorders in mitochondria and leads to the metabolic resulted from the changes in the patients with autism. This was recently shown by Subhadeep et al (2017), in a research on the comparison of the relative concentration of necessary fatty acids (linoleic and linolinic alpha), the long unsaturated fatty acids and phospholipids in the plasma of the patients with autism in Saudi Arabia with the control group of the same age. They reported the remarkable modulation of fatty acid metabolism that was evaluated by the change of the ratio among necessary fatty acids, long unsaturated fatty acids and omega 3, omega 6 fatty acids and the decrease of the circulation level of phospholipids. The authors have put a special emphasis on the phosphatidyl ethanol amine, phosphatydil serine and phosphatidyl lipid species that can be used as potential biological markers for the treatment strategies or future preventions (Subhadeep et al. 2017).

\section{MICROBIAL, PROBIOTIC AND ATOPIC DISEASES}

Human Skin Microbial and Atopic: Human skin contains several dominant bacterial species in different places and they are Propionibacterium, Eubacterium, Staphylococcus and Streptococcus and a fungoid species called Malaseziya. The investigations that focused on the particular parts of the body, have determined the important properties of colonization in healthy people. Corinobacterium is the most common bacterial species in anterior nasal cavities and Staphylococcus aureus pathogen exists in healthy people. Dermatitis atopic Staphylococcus that includes Staphylococcus aureus, Staphylococcus epidermidis, has an important role in worsening the disease. Unfortunately, for the patients, the identification of probiotic strains that may have useful effects on human skin has not been determined (Johnson et al. 2016).

Microbial Airways: Asthma and Atopic: The changes in the human microbial and pathogens have had influences as the possible reasons of asthma. In a research on the healthy infants and infants with asthma, there was no 
important change in the bacterial fila found in alimentary canal and the dominant fila in both groups having Bacteroides, Freiquito and Proteobacteria. While healthy infants were determined by the Streptococcus pre vetal, Veillonella and Fusobacterium. Haemophilus species asthmatic group was relatively abundant. In haemophilus species, pathogenic haemophilus influenza was considered as the asthma initiator. Although the future probiotic solutions may be applied by oral and inhaled indication, there might be gaps in our understanding of airway microbial, effective probiotics and their effects on the asthma and allergic diseases (Johnson et al. 2016).

The outbreak of atopic diseases such as exema, asthma and food allergies increases and it is related to the change of the intestinal microbiota. Health assumptions made by Strachan in 1989 showed that non-infection in early infancy causes the increase of atopic disease. Since the mutual effect of immune cells with microbial antigens is essential and important for the function and development of adaptive immune response, non-stimulation of immunity during the early life in the developed countries can cause the increase of immunity disorder which has been observed in asthma and atopic diseases.Delay or change in the main microbial can have an influence on the development of immunity response. Epidemiological data provide more evidence that shows the babies born by cesarean section, in comparison with the babies born through the vagina, develop atopic diseases such as asthma and type 1 diabetes and nutritional allergies more, (Versalovic et al. 2017).

\section{OBESITY AND ITS RELATION WITH MICROBES}

\section{Obesity Metabolic Syndrome}

The decrease of physical activity and the increase of energy consumption, particularly in the West, disrupts the energy balance in human beings and can lead to a complicated disease symptom that indicates in all a metabolic syndrome. The key characteristics of metabolic syndrome include obesity, the lack of glucose control, increased blood fat and blood pressure (Han and lean, 2016).

Intestinal Microbial Flora and Modulation of Inflammation: Obesity and insulin sensitivity are related to the enhanced modes of inflammatory symptoms in adipose tissue and the increase of pre-inflammatory cell level. It is getting more obvious that the low level of metabolic inflammation creates a causal relation between the obesity and insulin sensitivity. Human gut lumen acts as the source of LPS that is the main part of the outer membrane of gram negative bacteria. Several investigations have shown that the amount of this LPS in the blood of healthy people was obtainable; this indicates that LPS was continually absorbed by the intestine at low speeds. Interest- ingly, the circulation of LPS is related to the amount of insulin and in patients with type 2 diabetes, the amount of LPS circulation is higher. Sicard and et al, showed that there was a direct relation between the increase of LPS circulation and metabolic diseases and causes the increase of insulin sensitivity (Sicard et al. 2017).

Microbial Signaling through the Immune System: Alimentary canal has been covered with a number of epithelial cells which have a defensive role and is among the bacteria that are located in the alimentary canal lumen with under space; this secretory dam that consists of secreted and muscosal glycoprotein and black goblet cells is reinforced and pent cellulose cells cause the secretion of antimicrobial peptides such as lysozyme, spla2, RegIII $\beta$. The task of $M$ cells is sampling from the lumen space and they sample microbial antigens and send them to dendritic cell and they go towards the inflammatory and non-inflammatory responses and active $B$ cells for the secretion of IgA. All these factors cause the body to be in a balanced mode of its own microbiota and have a balanced mode of microbium in the stimulation of immune system (Spiljar et al. 2017).

Intestinal microbiota and some proteobiotics can increase the development of intestinal dam. They have changed some of the special species of probiotic expression of strong binding proteins including acladine, sinolin, cladin-2 and Z0-2 (Greenhalgh et al. 2016). The consumption of high-fat diet is also related to the increase of gram-negative bacteria than pram-positive. These findings are also the indication of this view that intestinal microbiota can be the change factor in the metabolic mode and lead to the endotoxemia and metabolic diseases. The treatment of the mice which have high-fat diet with antibiotic, decreases the normal amount of LPS plasma and as a result, the outbreak of the inflammation of adipose tissues, oxidative stress and macrophage markers decrease. Besides, it prevents from the hypertrophy of adipose cell and improves the metabolic parameters of diabetes and obesity in the mice with high-fat diet. Therefore, the fat in the diet can be related to the increase of LPS absorption and this occurs with changes in intestinal microbiota; therefore, the gram negative bacteria like Bacteriomate in Eubacterium, rectal Clostridium cocoides and also Bifidobacteria decrease (Petersen et al. 2017).

Gut Permeability and Metabolic Endotoxemia: High fat diet, increases the LPS plasma and is defined as "metabolic endotoxemia" and we found out that fat consumption changes the bacterial population (i.e. the decrease of Bifidobacterium) and finally, the fat consumption and obesity increase the gut permeability. We show that intestinal microbiota has an important role in the 


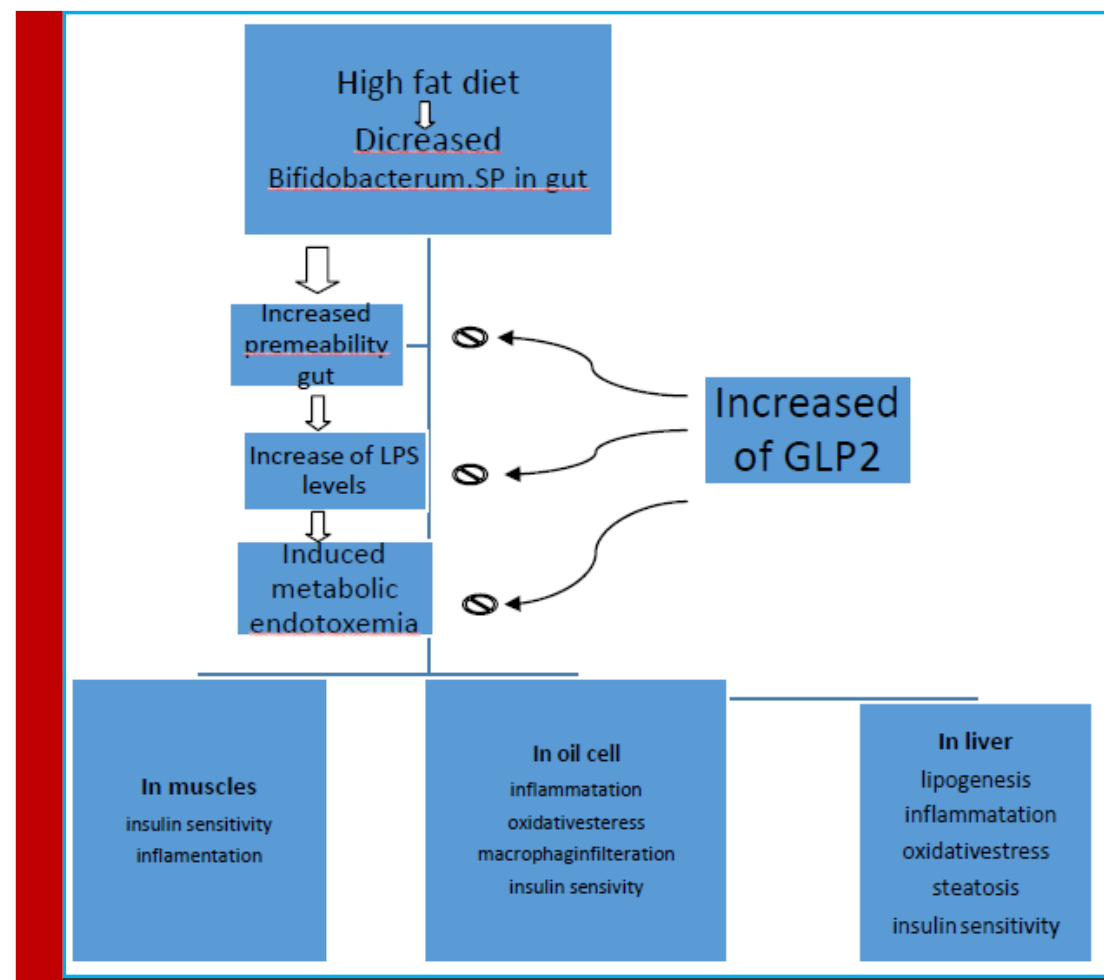

FIGURE 1. Changes in intestinal microbiota (after high-fat diet or obesity) improves the gut permeability, increases the metabolic endotoxemia and causes the development of metabolic disorder (Greenhalgh et al. 2016). For example, high-fat diet consumption changes the intestinal microbiota composition with a complicated method and the decrease of bifidobacterium spp. (Miliani et al. 2018) this phenomenon is accompanied by permeability that leads to the higher LPS levels of plasma (metabolic endotoxemia). (Petersen et al. 2017) Metabolic endotoxemia also increases the metabolic disorder resulted from low grade inflammation (insulin sensitivity, diabetes, obesity, steatosis, oxidative stress and adipose tissue macrophage infilteration. The increase of endogenous GLP-2 production causes the maintenance of bowel dam function, the decrease of metabolic endotoxemia and the development of metabolic disorder (Pflughoeft and Versalovic, 2012).

metabolic endotoxemia development and metabolic diseases along with obesity. For example, we found out that the remarkable changes in intestinal microbiota increase the insulin sensitivity completely through the antibiotic treatment, the related metabolic endotoxemia and metabolic disorders, i.e. glucose intolerance and insulin sensitivity (Haghes et al. 2017).

The various reports have shown that obesity because of diet manipulation (high-fat diet consumption) is determined by changes in intestinal microbiota towards the decrease of Bifidobacteria. In this research, in probiotic fed rats, the amount of intestinal Bifidobacteria was maintained and the effect of metabolic endotoxemia resulted from high-fat diet and inflammatory disorders, decreased. We have recently found out that the intestinal microbiota modulation has controlled and increased the production of intestinal inotropic peptide endogenous, i.e. glucagon-like peptide 2 (GLP-2) and subsequently, has improved the bowel dam function by a GLP-2 based mechanism (figure 1,2).

Contagious and Cardiovascular diseases: From the early half of the $19^{\text {th }}$. Century, contagious diseases have been known as the factor of artery hardening that with the changes including the increase of cholesterol absorption and lipoprotein improved by low density and the increase of the pressure of adhesion molecules and inflammatory cytokines lead to the vulnerability of the blood plaques along with the artery hardening. Epidemiological investigations have shown that there is a relationship among the inflammation of the gum, chlamydia pneumonia and contagion of helix pylori bacteria and the hardening or arteries. Indeed, chlamydia pneumonia can lead to the formation of sponge cells in blood vessel walls and the 


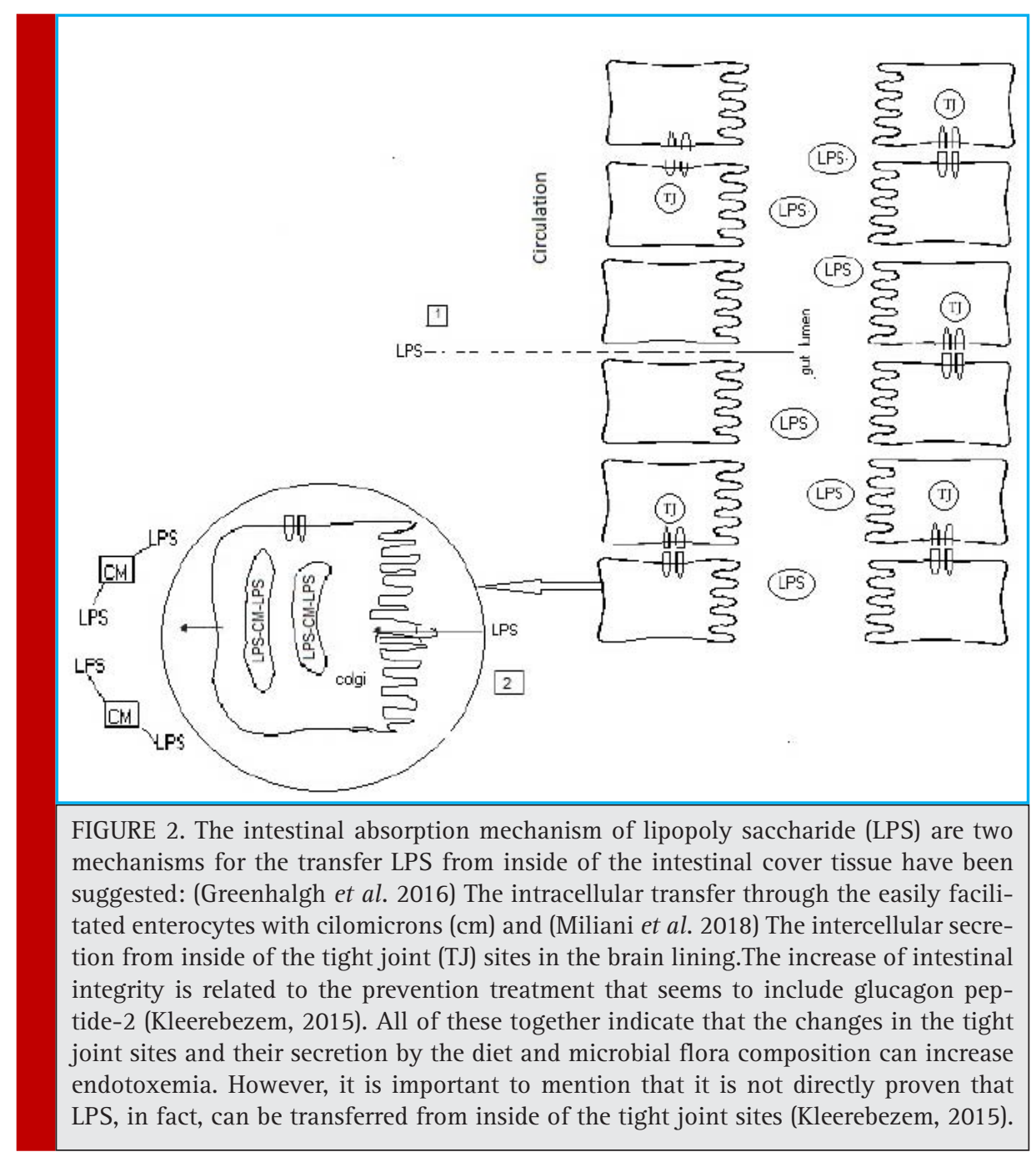

bacterial DNA can be found in 50\% of blood plaques. It is possible that chlamydia pneumonia can infect the macrophages and lymphocytes in the lungs and move to aortic vascular walls through the cells. Since there is a close relation between cardio-vascular disease and contagious disease, attempts have been made to treat the hardening of arteries by antibiotics (Kleerebezem, 2015). The conflict of inflammatory paths and metabolic in the hardening of arteries. The activation of LXR in macrophages lead to the cholesterol efflux from the cell and the activation of TLR4 by bacterial LRS or OXLDL, however, prevents LXRs and stimulates metalloproteinase (MMP4), (Kleerebezem, 2015).

Oral and Intestinal Natural Microbial Flora and Hardening of Arteries: The incomplete health of the teeth is related to the increase of the blockage of heart vessels and the result of several investigations show that there is an oral source for the bacterial plaque. To confirm these findings, oral pathogen can often be found in plaques and the direct control of P.gingivalis shows that this bacterium can lead to the increase of artery hardening. Insufficient oral sanitation has been recently attributed to the cardiovascular damages. Interestingly, it is related to the inflammation low intensity that is related to the cardiovascular advances. This information shows that bacterial oral cavity can increase the inflammations with low intensity and cause the hardening of arteries, (Kleerebezem, 2015).

\section{PROBIOTICS AND PREBIOTICS}

Probiotics and prebiotics may be useful in keeping the suitable microbial balance of intestinal microbiota of the elderly and help decrease the harmful effects of antibiotic consumption and less nutrition. Probiotics are alive microbes and when they are used in sufficient amounts, they are useful for the host. Prebiotics are the indigestible ingredients including various inulins and oligosaccharides that stimulate selectively the growth 
of bacterial population in colon. Bifidobacterium and lactobacillus in the elderly often decrease with the disruption of health (Amy et al. 2015).The investigations show that probiotic consumption that includes these strains can increase their abundance remarkably along with the decrease of pathogenic micro-organisms in the intestine. A recent investigation in the lab environment showed that the intestinal microbiota of the elderly can be improved by suitable probiotic. Bifidobacterium and lactobacillus species along with the two prebiotics were added to the stool culture of the elderly participants. The addition of useful bacteria increases Bifidobacteria and decreases Bacteroides. The probiotic-prebiotic composition also increases the amount of Bifidobacterium and Lactobacillus. These all show that the main change in the intestinal microbiota for colon is healthier. Of course, prebiotic can alone improve health and change the intestinal microbial composition of healthy population (Caesar et al. 2016).

\section{REFERENCES}

Amy L, Nathan C, Gautam D. (2015): The effects of antibiotics on the microbiome throughout development and alternative approaches for therapeutic modulation. Genome Med. doi: 10.1186/s 13073-016-0294-z.

Arboleya S, Watkins C, Stanton C, Ross RP. (2016): Gut Bifido bacteria Populations in Human Health and Aging. Front. Microbiol. https://doi.org/10.3389/fmicb.2016.01204.

Bailey M.T, Lubach G.R, Coe C.L. (2015). Prenatal stress alters bacterial colonization of the gut in infant monkeys. J Pediatr Gastroenterol. Nutr 38: 414-421.

Bölte S, Bartl-Pokorny KD, Jonsson U, Berggren S, Zhang D, Kostrzewa E, Falck-Ytter T, Einspieler C, Pokorny FB, Jones EJ, Roeyers H, Charman T, Marschik PB. (2016). How can clinicians detect and treat autism early? Methodological trends of technology use in research. Acta paediatrica 105 (2): 137-144.

Caesar R, Nygren H, Orešič M, Bäckhed F. (2016). Interaction between dietary lipids and gut microbiota regulates hepatic cholesterol metabolism. J Lipid Res. 57(3):474-81. doi: 10.1194/ jlr.M065847.

Dinan TG, Cryan JF. (2017). The Microbiome-Gut-Brain Axis in Health and Disease. Gastroenterol Clin North Am. 46(1):77-89. doi: 10.1016/j.gtc.2016.09.007.

Dominianni C. (2016): The gut microbiota in conventional and serrated precursors of colorectal cancer. Microbiome (4:69). https://doi.org/10.1186/s40168-016-0218-6.

Greenhalgh K, Meyer K.M, Aagaard K, Wilmes. (2016). The human gut microbiome in health: establishment and resilience of microbiota over a life time. Envionment microbiology.18 (7): 2103-2116. DOI: 10.1111/1462-2920.13318 View/save citation.
Han TS, Lean ME. (2016). A clinical perspective of obesity, metabolic syndrome and cardiovascular disease. JRSM Cardiovasc Dis. doi: 10.1177/204800401663337.

Hughes K, Harnisch L, Alcon-Giner C, Mitra S, Wright S, Ketskemety L, van Sinderen D, Watson A, Hall L. (2017). Bifidobacterium breve reduces apoptotic epithelial cell shedding in an exopolysaccharide and MyD88-dependent manner. Open Biol. 7(1): 160155. doi: 10.1098/rsob.160155.

Johnson CH, Spilker ME, Goetz L, Peterson SN, Siuzdak G. (2016). Metabolite and Microbiome Interplay in Cancer Immunotherapy. Cancer Res. 1:76(21):6146-6152. Epub 2016 Oct 11.

Kleerebezem M. (2015). Microbial ecosystem management: strategies to adapt ecosystems to improve performance and health impact. Current Opinion in Biotechnology.l (32), pp.vviii [Peer Reviewed Journal].

Milaini C, Turroni F, Duranti S, Lugli GA, Mancabelli L, Ferrario C, van Sinderen D, Ventura M AEM. (2018). Genomics of the Genus Bifidobacterium Reveals Species-Specific Adaptation to the Glycan-Rich Gut Environment 84(5).

Petersen L, Bautista E, Nguyen H, Hanson B, Chen L, Lek S, Sodergren E, Weinstock G. (2017): Community characteristics of the gut microbiomes of competitive cyclists. Microbiome. 5(98).https://doi.org/10.1186/s40168-017-0320-4.

Sicard J, Bihan G, Vogeleer P, Jacques M, Harel J. (2017). Interactions of Intestinal Bacteria with Components of the Intestinal Mucus. Front. Cell. Infect. Microbiol. https://doi.org/10.3389/ fcimb.2017.00387.

Spiljar M, Merkler D, Trajkovski M. (2017). The Immune System Bridges the Gut Microbiota with Systemic Energy Homeostasis: Focus on TLRs, Mucosal Barrier, and SCFAs. Front Immunol. 8: 1353.doi: 10.3389/fimmu.2017.01353.

Subhadeep R, Atul Kumar R, Shreesh R, Uma D, Manjari S, Swetlana G, Rajnish K, Jitendra K, Lakhveer S, Nazam A, Abdulaziz S, Rakesh P, Dinesh K. (2017). Alpha-linolenic acid stabilizes HIF-1 $\alpha$ and downregulates FASN to promote mitochondrial apoptosis for mammary gland chemoprevention. Oncotarget. 8(41): 70049-70071. doi: 10.18632/oncotarget.19551.

Timothy L, Zisman D, Damman J. (2016). The intestinal microbiome, barrier function, and immune system in inflammatory bowel disease: a tripartite pathophysiological circuit with implications for new therapeutic directions. Therap Adv Gastroenterol. 9(4): 606-625. doi: 10.1177/1756283X16644242.

Versalovic J, Dore J, Guarner F, Luna RA, Ringel Y. (2017). Microbiome-Based Diagnostics: Ready for Applications in Laboratory Medicine? Clin Chem. 2017 Nov;63(11):1674-1679. doi: 10.1373/clinchem.2016.264473.

Vinke P, El Aidy S, van Dijk G. (2017): The Role of Supplemental Complex Dietary Carbohydrates and Gut Microbiota in Promoting Cardiometabolic and Immunological Health in Obesity: Lessons from Healthy Non-Obese Individuals. Front Nutr. doi: 10.3389/fnut.2017.00034. 